Abanico Veterinario. Enero-Diciembre 2021; 11:1-9. http://dx.doi.org/10.21929/abavet2021.7

Nota Corta. Recibido: 03/04/2020. Aceptado: 18/12/2020. Publicado: 06/02/2021. Clave: 2020-15.

\title{
Microorganismos de montaña y ensilado de maíz como probióticos en la engorda de conejos
}

Mountain microorganisms and corn silage as probiotics in the fattening of rabbits

\section{Medina-Saavedra Tarsicio*1 ID , Dzul-Cauich Jorge ${ }^{1 \text { ID }}$, Arroyo-Figueroa Gabriela ${ }^{1 \mathrm{ID}}$, García-Vieyra Isabel ${ }^{1 \text { ID }}$, Quiñones-Páramo Mónica1 ${ }^{\text {ID }}$, Mexicano-Santoyo Lilia ${ }^{1 \text { ID }}$}

1 Universidad de Guanajuato, Campus Celaya Salvatierra, División de Ciencias de la Salud e Ingeniería, Departamento de Ingeniería Agroindustrial, Salvatierra Guanajuato México. *Autor responsable y de correspondencia Medina-Saavedra Tarsicio. El Mayorazgo, Ignacio Zaragoza No. 749, Centro. C.P 38900 Salvatierra, Guanajuato México. tarsicioms@hotmail.com, jorge.dzul@ugto.mx, gabiaf@yahoo.com.mx, isagarvi26@gmail.com,moni_ni21@hotmail.com, lilia_lasalle@hotmail.com.

\section{RESUMEN}

Existen bacterias que producen ácido láctico (BAL) presentes en la microflora epifita de los vegetales, y consorcios de microorganismos de montaña como levaduras y cultivos mixtos que pueden ser empleados como probióticos y promotores del crecimiento en la producción animal. Para evaluar el uso de microorganismos de montaña en ensilado de maíz como probióticos en la engorda de conejos durante 4 semanas, se emplearon 20 conejos híbridos. Los cuales se escogieron aleatoriamente en cada tratamiento. La preparación de los probióticos se realizó mediante una etapa de fermentación inicial anaerobia y una final aerobia. El tratamiento (T1) fue una dieta suplementada con la adición de microorganismos de montaña en ensilado de maíz (MME) en el agua de bebida y el tratamiento dos o convencional (T2) fungió como testigo sin aplicación de MME. Se calculó el consumo de alimento y el indicie de conversión alimenticia, expresados como la media \pm la desviación estándar. Al realizar un análisis de varianza (ANOVA) se estableció que durante la semana 4 del tratamiento hubo diferencia significativa $(P<0.05)$ en la ganancia de peso y conversión alimenticia entre los tratamientos, siendo favorable para T1 suplementados con MME.

Palabras Clave: Ensilaje, microorganismos eficientes, cunicultura, probióticos.

\begin{abstract}
There are bacteria that produce lactic acid (LAB) present in the epiphytic microflora of plants, and consortia of mountain microorganisms such as yeasts and mixed cultures that can be used as probiotics and growth promoters in animal production. To evaluate the use of mountain microorganisms in corn silage as probiotics in the fattening of rabbits for 4 weeks, 20 hybrid rabbits were used. Which were chosen randomly in each treatment. The preparation of the probiotics was carried out through an initial anaerobic fermentation stage and a final aerobic one. Treatment (T1) was a diet supplemented with the addition of mountain microorganisms in corn silage (MME) in the drinking water and treatment two or conventional (T2) served as a control without application of MME. The feed consumption and the feed conversion index were calculated, expressed as the mean \pm the standard deviation. When performing an analysis of variance (ANOVA), it was established that during week 4 of treatment there was a significant difference $(P<0.05)$ in weight gain and feed conversion between treatments, being favorable for T1 supplemented with MME.
\end{abstract}

Keywords: Silage, efficient microorganisms, cuniculture, probiotics. 


\section{INTRODUCCIÓN}

Los microorganismos de montaña (MM) también llamados microorganismos benéficos, se encuentran presentes en los ecosistemas naturales poco afectados por factores antrópicos, en donde se han identificado bacterias fotosintéticas, bacterias ácido-lácticas (BAL), levaduras y actinomicetos (Campo et al., 2014; Ramírez et al., 2016), que al desarrollarse en una adecuada cantidad de materia orgánica secretan sustancias benéficas que inhiben o controlan el crecimiento de las poblaciones de microorganismos patógenos (Cóndor et al., 2007).

Los géneros Lactobacillus, Pediococcus, Leuconostoc, Enterococcus, Lactococcus y Streptococcus, producen Ácido Láctico (BAL) y se encuentran presentes en la microflora epifítica de los vegetales (Garcés et al., 2004). Por otro lado, el género Bacillus, proveniente del contenido gastrointestinal de diversas especies animales o de sus heces, es el más utilizado como probióticos y promotores del crecimiento en la producción animal (Sánchez et al., 2015). Los Bacillus spp son bacterias aerobias facultativas que fermentan un amplio rango de carbohidratos y son utilizadas para inhibir el proceso de deterioro aeróbico en ensilajes debido a que tienen la capacidad de producir sustancias fungicidas, no obstante, son menos eficaces como productores de ácido láctico y acético en comparación con las BAL (Garcés et al., 2004; Layton et al., 2011).

Por otra parte, el ensilaje es el proceso de fermentación de los carbohidratos solubles del forraje por medio de bacterias que producen ácido láctico en condiciones anaeróbicas (Garcés et al., 2004). Mediante la fermentación anaerobia controlada, se mantiene estable la composición del material ensilado durante largo tiempo a través de la acidificación del material utilizado, minimizando asi, las fermentaciones secundarias indeseables como la fermentación alcohólica, producida por las levaduras, que son un peligro de toxicidad para el ganado y la fermentación butírica producida por el género Clostridium (Garcés et al., 2004).

Para que exista una fermentación óptima y controlada es necesaria la adecuada proporción entre las bacterias lácticas y los carbohidratos solubles; se pueden emplear diferentes aditivos para inducir y optimizar el proceso fermentativo, como la melaza, la pulpa de cítricos o el maíz triturado, que proveen una fuente de azúcares solubles que la bacteria utiliza para producir ácido láctico, estabilizando así el medio (Valencia et al., 2011). El proceso de ensilaje no mejora la calidad del forraje, solo conserva su valor nutricional, como los componentes energeticos y proteicos mediante procesos de fermentación y manteniendolo estable por mucho tiempo (Villa y Hurtado, 2016).

El uso de los probióticos contribuye al equilibrio microbiano intestinal, estimulando el sistema inmunológico del animal, produciendo ácidos orgánicos, bacteriocinas y enzimas que favorecen a la absorción de los nutrientes, mejorando los parámetros productivos (Gutiérrez et al., 2014). 
Mucho se ha publicado acerca de los probióticos y sus diferentes efectos, en el caso de la producción de conejos, no obstante, la garantía de éxito en conejos está dada por los resultados productivos esperados. Los conejos se caracterizan por su facilidad de manejo, rápida reproducción y la obtención de una proteína animal de calidad, que sitúa a la cunicultura en un lugar que favorece la producción a pequeña y mediana escala. La carne de conejo representa grandes ventajas, con un balance adecuado de ácidos grasos, proteína, vitaminas y minerales, bajo contenido en colesterol y sodio (Para et al., 2015).

De acuerdo con datos del Instituto Nacional de Estadística, Geografía e Informática (INEGI) los estados con mayor producción de conejos son Hidalgo, Puebla, Tlaxcala, Estado de México y Guanajuato. El desarrollo de la cunicultura en México se ve limitada por la falta de apoyo oficial, aunado a un manejo inadecuado de la sanidad y la nutrición, además la poca promoción de los beneficios de la carne de conejo, reflejado en un bajo consumo per cápita, de entre 38 y 134 g durante 2008 y 2009 (Armada, 2016), a pesar de las grandes ventajas desde el punto de vista nutricional (Coreno et al., 2017). E objetivo del presente trabajo fue evaluar el uso de microorganismos de montaña en ensilado de maíz como probióticos en la engorda de los conejos.

\section{MATERIAL Y MÉTODOS}

El presente estudio se realizó en la Sede Salvatierra de la Universidad de Guanajuato, ubicada en Salvatierra, Guanajuato, México $\left(20^{\circ} 12^{\prime} 45.51 " \mathrm{~N}, 100^{\circ} 52^{\prime} 30.09\right.$ "O, a 1,749 msnm) (Google Earth, s.f). El experimento fue realizado con conejos híbridos de la raza california de 30 días de edad y peso similar. Cada uno fue alojado en jaulas individuales provistas de bebedero y comedero. El experimento tuvo una duración de cuatro semanas.

\section{Preparación del ensilado de maíz con microorganismos de montaña}

Los microorganismos de montaña en ensilado de maíz (MME) se obtuvieron de un proceso realizado en tres etapas, las dos primeras de manera sólida anaerobia y la tercera de forma líquida y aerobia. Durante la etapa uno, se recolectaron los microorganismos de montaña (MM) mediante la hojarasca de un sitio ecológico con poca afectación antrópica ubicado cerca de la Ciudad de Salvatierra Guanajuato y se le incorporó $10 \%$ de harina de maíz, $5 \%$ de melaza, reservándolo por 30 días en un recipiente de plástico con capacidad de 20 litros y la tapa se selló de manera que no entrara oxígeno.

Durante la segunda etapa se tomó producto resultado del proceso anterior y se mezcló con la misma cantidad de ensilado de maíz, además de $10 \%$ de harina de maíz y $5 \%$ de melaza, lo que se guardó durante 30 días, de manera anaerobia. Finalmente, la tercera etapa consistió en tomar $500 \mathrm{~g}$ del producto resultado de la etapa 2 y se envolvió en una manta y se colocó en un recipiente de 20 litros que contenía agua no clorada adicionada 
con el $1 \%$ de melaza y durante 72 horas se proporcionó aireación para posteriormente guardarse en recipientes de plástico.

\section{Recuento de microorganismos totales}

Para determinar el número de microorganismos totales presentes en la fase líquida de los MME a las 24, 48 y $72 \mathrm{~h}$, se tomaron muestras de $100 \mu \mathrm{L}$ y se realizaron diluciones seriadas. Posteriormente, en cajas Petri que contenían medio sólido agar dextrosa papa, se inocularon $100 \mu \mathrm{L}$ de las diluciones 1:100000 y 1:1000000 (cada uno de los procedimientos antes mencionados se realizó por triplicado). Las cajas inoculadas se incubaron en una estufa de cultivo marca Terbaf ${ }^{\mathrm{MR}}$ a $30^{\circ} \mathrm{C}$ durante $48 \mathrm{~h}$, transcurrido el tiempo de incubación, se contaron las colonias en cada una de las cajas y se calcularon las unidades formadoras de colonias por mililitro (UFC $/ \mathrm{mL}$ ).

\section{Tratamientos}

Los tratamientos fueron aplicados a 20 conejos híbridos de la raza california de 30 días de edad, los cuales se distribuyeron aleatoriamente en dos grupos. Los conejos fueron alimentados con una dieta suplementada con $10^{7} \mathrm{UFC} / \mathrm{mL}$ de MME en el agua de bebida y alimento comercial (T1). El tratamiento dos (T2), que fungió como control, consistió en una dieta convencional sin suplemento probiótico (MME) en el agua de bebida y alimento comercial. En ambos tratamientos, el alimento y el agua se proporcionaron ad libitum. Diariamente se cambió el agua de bebida que no consumían. El consumo de alimento se calculó con la siguiente formula:

Donde:

$$
A c=(A o-A r)
$$

Ac=Alimento consumido

Ao=Alimento ofrecido

Ar=Alimento rechazado

Se registró el incremento de peso semanal de los conejos realizando el pesaje al inicio de la prueba y durante cuatro semanas, calculando la diferencia entre el peso vivo actual y el peso vivo de la semana anterior.

Se determinó el índice de conversión alimenticia (IC) mediante la siguiente fórmula:

$$
I C=(A C S / G P S)
$$

Donde:

$\mathrm{IC}=$ Índice de conversión alimenticia

ACS = Alimento consumido semanal $(\mathrm{kg})$

GPS $=$ Ganancia de peso semanal $(\mathrm{Kg})$ 
Los datos obtenidos de ganancia de peso y conversión alimenticia fueron expresados como la media \pm la desviación estándar, además se realizó un análisis de varianza (ANOVA) utilizando el programa Statgraphics Centurión (Statgraphics.Net, 2021).

\section{RESULTADOS Y DISCUSIÓN}

El número de microorganismos totales presentes en la fase líquida de MME expresado en UFC/ $\mathrm{mL}$ se muestran en la tabla 1 . Los resultados muestran un incremento en el número de microorganismos a medida que transcurre el tiempo, obteniendo un recuento total de $9 \times 10^{7} \mathrm{UFC} / \mathrm{mL}$ en un periodo de 72 horas.

Tabla 1. Microorganismos totales presentes en la fase líquida

\begin{tabular}{cc}
\hline Tiempo (horas) & UFC/ mL \\
\hline 24 & $3 \times 10^{6} \pm 2.0^{\mathrm{a}}$ \\
48 & $4 \times 10^{7} \pm 2.8^{\mathrm{b}}$ \\
72 & $9 \times 10^{7} \pm 2.5^{\mathrm{c}}$
\end{tabular}

Guo et al. (2017), al suplementar con $10^{5}, 10^{6}$ y $10^{7} \mathrm{UFC} / \mathrm{g}$ de Bacillus subtilis la dieta de conejos, observaron un mejor rendimiento en peso con la suplementación de $10^{6} \mathrm{UFC} /$ g. Por otra parte, Phuoc y Jamikorn (2017) suplementaron la dieta de conejos con 1X 10 UFC/ $g$ de $B$. subtilis, $1 \times 10^{7} \mathrm{UFC/} \mathrm{g}$ de L. acidophilus o la combinación de ellos a una concentración de $0.5 \times 10^{7} \mathrm{UFC} / \mathrm{g}$, sugiriendo que la suplementación de la dieta con estos microorganismos tiene beneficios como probióticos en los conejos. En el presente trabajo se obtuvo un número total de $9 \times 10^{7} \mathrm{UFC} / \mathrm{mL}$, cantidad de microorganismos similares a los trabajos antes mencionados y que pueden tener un potencial probiótico al ser suministrado en agua de bebida de los conejos.

Los resultados de ganancia de peso y conversión alimenticia se presentan en la tabla 2. Estadísticamente no se observan diferencias significativas entre los dos tratamientos con respecto a la ganancia de peso; sin embargo, se puede notar que, en relación con la conversión alimenticia, existen diferencias significativas entre los tratamientos, siendo T1 el tratamiento con mayor conversión alimenticia $5.65 \pm 0.6$. Gutiérrez et al. (2014), alimentaron cerdos con microorganismos probióticos nativos reportando que no hubo diferencia estadística significativa en la ganancia de peso y en relación con la conversión alimenticia y que el tratamiento que tuvo el mejor perfil de conversión fue al que no se le adicionó microorganismos probióticos.

De acuerdo con Villa y Hurtado (2016) los conejos alimentados con ensilajes obtienen la mayor ganancia de peso, que los animales alimentados con solo forrajes frescos, dentro de las bacterias ácido lácticas presentes en el ensilado y los MM se encuentran los géneros Lactobacillus, Streptococcus y Bifidobacterium, los cuales producen ácidos 
orgánicos, bacteriocinas, preservantes, vitaminas, endulzantes, aromatizantes, sabores, antioxidantes, entre otros (Parra, 2010) que contribuyen a mejorar la producción animal, en relación a parámetros de cantidad y/o composición de la leche, condición corporal, ganancia de peso vivo y desarrollo reproductivo (Phipps et al. 2000).

Tabla 2. Ganancia promedio de peso y conversión alimenticia durante el tiempo de evaluación (4 semanas).

\begin{tabular}{ccc}
\hline Tratamiento & $\begin{array}{c}\text { Ganancia promedio de peso } \\
(\mathrm{kg})\end{array}$ & Conversión alimenticia \\
\hline T1 & $0.163 \pm 0.03^{\mathrm{a}}$ & $5.65 \pm 0.6^{\mathrm{a}}$ \\
$\mathrm{T} 2$ & $0.198 \pm 0.04^{\mathrm{a}}$ & $3.76 \pm 0.6^{\mathrm{b}}$ \\
\hline
\end{tabular}

En las figuras 1 y 2 , se pueden observar la variabilidad de los tratamientos. En la figura 1 se puede observar que la máxima ganancia de peso en T1, fue de $0.211 \mathrm{~kg}$ y la mínima fue de $0.135 \mathrm{~kg}$, en relación con el tratamiento 2, la máxima ganancia de peso a lo largo del tiempo de evaluación fue de $0.262 \mathrm{~kg}$ y la mínima fue de $0.167 \mathrm{~kg}$. En la figura $2 \mathrm{se}$ puede ver la variabilidad de la conversión alimenticia en los conejos tratados con y sin microrganismos de montaña. La máxima conversión alimenticia fue de 6.22 y la mínima fue de 4.8 para el tratamiento 1, mientras que para el tratamiento 2 la máxima conversión alimenticia fue de 4.2 y la mínima de 2.9.

Gráfico Caja y Bigotes

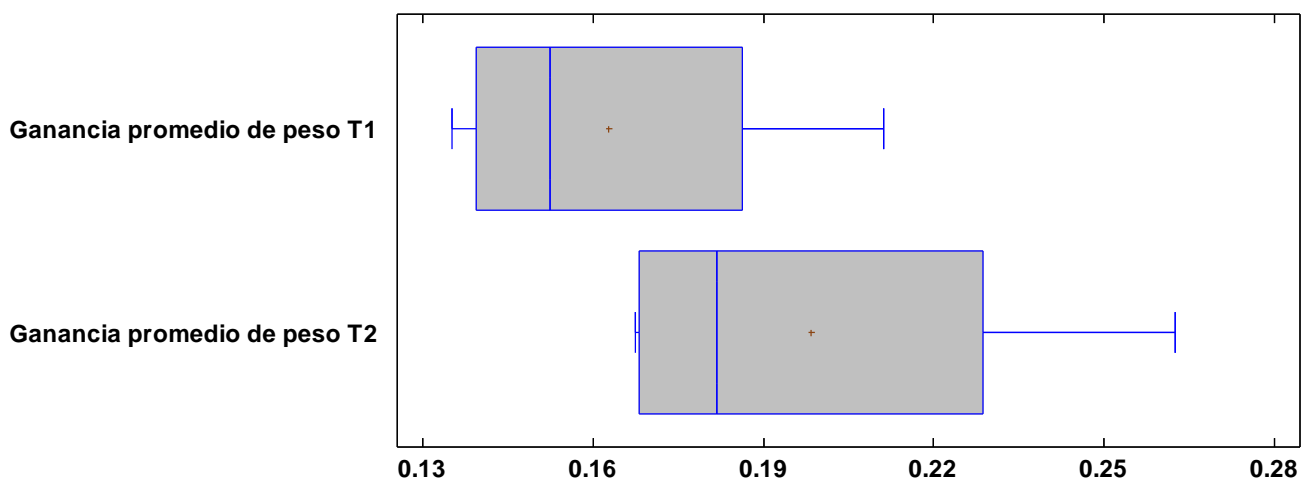

Figura 1. Distribución de los pesos promedio en conejos tratados con y sin microorganismos de montaña 
Grafico Caja y Bigotes

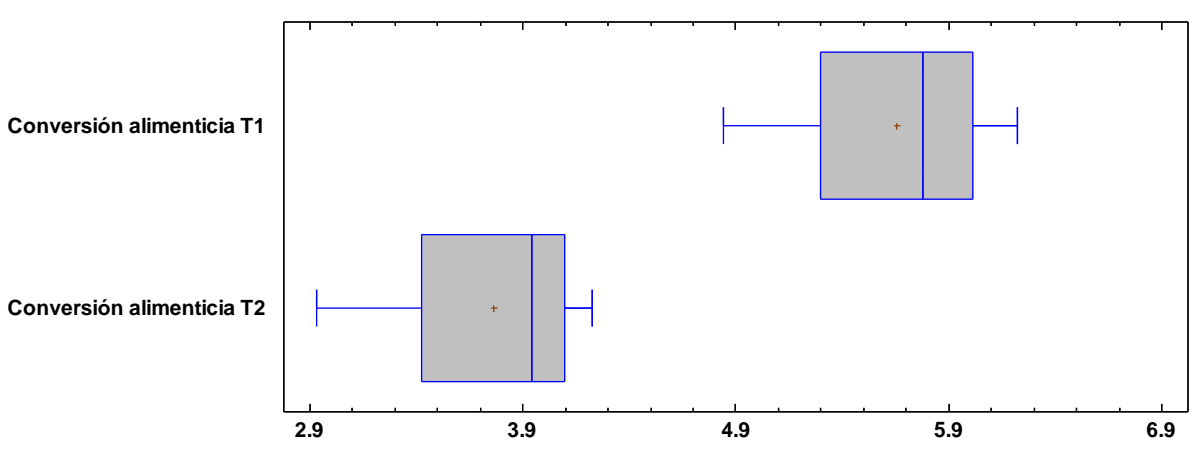

Figura 2. Distribución de los promedios de conversión alimenticia en conejos tratados con y sin microorganismos de montaña

\section{CONCLUSIÓN}

Los microorganismos de montaña obtenidos de ecosistemas poco afectados de forma antropogénica son una fuente de bacterias productoras de ácido láctico, que pueden mejorar el valor nutricional del ensilaje y ser utilizados como probióticos en al agua de bebida, incrementando el rendimiento en el proceso de engorda del conejo, con el fin estimular el desarrollo de las técnicas de cunicultura en México, y sugerir métodos de caracterización de microorganismos productores de ácido láctico.

\section{LITERATURA CITADA}

ARMADA ER. 2016. La explotación cunícola en México, una revisión a través del VIII Censo Agrícola, Ganadero y Forestal 2007.

http://www.ancum.com.mx/web/pdfs/Organizacion\%20de\%20productores/LA\%20EXPL OTACION\%20CUNICOLA\%20EN\%20MEXICO.pdf

CAMPO MAP, Acosta SRL, Morales VS, Prado FA. 2014. Evaluación de microrganismos de montaña $(\mathrm{mm})$ en la producción de acelga en la meseta de Popayán. Biotecnología en el Sector Agropecuario y Agroindustrial. 12(1):79-87. http://www.scielo.org.co/pdf/bsaa/v12n1/v12n1a10.pdf

CÓNDOR AF, Gonzáles P, Lokare C. 2007. Effective Microorganisms: Myth or reality? Revista Peruana de Biología. 14 (2): 315-319. http://www.scielo.org.pe/pdf/rpb/v14n2/a26v14n02 
CORENO HO, García VS, Ayala MM, Soto SS, Ojeda RD, Zepeda BA. 2017. Efecto del consumo de vinagre y una bebida fermentada sobre la calidad de la canal y carne de conejos. Abanico veterinario. 7(1):48-52. https://dx.doi.org/10.21929/abavet2017.71.5

GARCÉS AM, Berrio LR, Ruiz SA, Serna JD, Buile A. 2004. Ensilaje como fuente de alimentación para el ganado. Revista Lasallista de Investigación. 1(1): 66-71. https://www.redalyc.org/articulo.oa?id=695/69511010

GOOGLE Earth, s.f. [Mapa de El Mayorazgo, Salvatierra Guanajuato, México. En Google Earth].

https://earth.google.com/web/search/Mayorazgo,+Zona+Centro,+Salvatierra,+Gto./@20. 2141147

100.87557165,1760.16647678a,984.14770726d,35y,0h,0t,0r/data=CnMaSRJDCiQweD g0MmNjNDkxMTg1M2E5NmQ6MHhkZmFkYWRINjJhZTc5ZGUZHd4Yp8Y2NEAhQhKs YAo4WcAqCU1heW9yYXpnbxgCIAEiJgokCdroYox3PTRAEX5LksVLHjRAGU6bB_MfM FnAlf4zW5HhP1nA

GUO M, Wu F, Hao G, Qi Q, Li R, Li N, Chai, T. 2017. Bacillus subtilis improves immunity and disease resistance in rabbits. Frontiers in Immunology. 8:354-354. https://dx.doi.org/10.3389/fimmu.2017.00354

GUTIÉRREZ RLA, Bedoya O, Ríos EM. 2014. Evaluación de parámetros productivos en cerdos (Sus scrofa domesticus) suplementados con microorganismos probióticos nativos. Journal of agriculture and animal scienses. 3(2): 48-58. http://repository.lasallista.edu.co:8080/ojs/index.php/jals/article/view/733

HEREDIA MF. 2017. Caracterización de microrganismos de montaña en biofertilizantes artesanales. Escuela Agrícola Panamericana, Zamorano. Obtenido de https://bdigital.zamorano.edu/bitstream/11036/6199/1/IAD-2017-049.pdf

LAYTON C, Maldonado E, Monroy L, Corrales L, Sánchez L. 2011. Bacillus spp.; perspectiva de su efecto biocontrolador mediante antibiosis en cultivos afectados por fitopatógenos. NOVA - Publicación Científica en Ciencias Biomédicas. 9 (15):177 - 187. https://dx.doi.org/10.22490/24629448.501

PARA PA, Ganguly S, Wakchaure R, Sharma R, Mahajan T, Praveen PK. 2015. Rabbit meat has the potential of being a possible alternative to other meats as a protein source: A brief review. Int $J$ Phar Biomedi Res. 2: 17-19. ISSN: 2394 - 3726. https://www.researchgate.net/publication/289674478 
PARRA RA. 2010. Bacterias ácido lácticas: Papel funcional en los alimentos. Revista de la Facultad de Ciencias Agropecuarias de la Universidad Pedagógica y Tecnológica de Colombia. 8 (1): 93-100. http://www.scielo.org.co/pdf/bsaa/v8n1/v8n1a12.pdf

PHIPPS RH, Sutton JD, Beever D. E.and A. K. Jones. 2000. The effect of crop maturity on the nutritional value of maize silage for lactating dairy cows. Animal Science. 71:401409.

https://www.researchgate.net/profile/Subha_Ganguly/publication/289674478_Rabbit_Me at_has_the_Potential_of_Being_a_Possible_Alternative_to_Other_Meats_as_a_Protein _Source_A_Brief_Review/links/5691deeb08ae0f920dcb9274.df

PHUOC TL, Jamikorn U. 2017. Effects of probiotic supplement (Bacillus subtilis and Lactobacillus acidophilus) on feed efficiency, growth performance, and microbial population of weaning rabbits. Asian-Australasian Journal of Animal Sciences. 30(2):198205. https://dx.doi.org/10.5713/ajas. 15.0823

RAMÍREZ HQ, Cadillo WT, Morales JJ. 2016. Evaluación de la calidad de un abono líquido producido vía fermentación homoláctica de heces de alpaca. Ecología Aplicada. 15(2):133-142. https://dx.doi.org/10.21704/rea.v15i2.753

STATGRAPHICS.NET. (18 de enero de 2021). Statgraphics.Net. Obtenido de Statgraphics.Net: https://statgraphics.net/

SÁNCHEZ MT, Ruiz MA, Morales ME. 2015. Microorganismos probióticos y salud. Ars Pharmaceutica. 56(1):45-59. https://dx.doi.org/10.4321/S2340-98942015000100007

SIMON O, Jadamus A, Vahjen W. 2001. Probiotic feed additives-effectiveness and expected modes ofaction. J Anim Feed Sci. 10: 51- 67. https://doi.org/10.22358/jafs/70012/2001

VALENCIA CA, Hernández BA, López DL. 2011. El ensilaje: ¿qué es y para qué sirve? La Ciencia $y$ el Hombre. 24(2): 1-14. https://www.uv.mx/cienciahombre/revistae/vol24num2/articulos/ensilaje/

VILLA RR, Hurtado J. 2016. Evaluación nutricional de diferentes ensilajes para alimentar conejos. Revista de Ciencias agrícolas. 33(2):76-83. https://doi.org/10.22267/rcia.163302.54 